\title{
Subjective ambiguity and preference for flexibility
}

\author{
Leandro Gorno ${ }^{\mathrm{a}}$, Paulo Natenzon ${ }^{\mathrm{b}, *}$ \\ ${ }^{\text {a } F G V ~ E P G E ~ E s c o l a ~ B r a s i l e i r a ~ d e ~ E c o n o m i a ~ e ~ F i n a n c ̧ a s, ~ R i o ~ d e ~ J a n e i r o, ~ B r a z i l ~}$ \\ ${ }^{\mathrm{b}}$ Department of Economics, Washington University in St. Louis, USA
}

\section{A R T I C L E I N F O}

\section{Article history:}

Received 10 July 2018

Revised 17 July 2018

Accepted 18 July 2018

Available online 16 August 2018

\section{Keywords:}

Preference for flexibility

Pessimism

Subjective ambiguity

Self-paternalism

\begin{abstract}
A B S T R A C T
A preference over menus is monotonic when every menu is at least as good as any of its subsets. We show that every utility representation for a monotonic preference is equal to the minmax value of a decision maker whose payoff depends on the option chosen from the menu and on the realization of a subjective state. This representation suggests a decision maker who faces uncertainty about her own future tastes and who exhibits an extreme form of pessimism with respect to this uncertainty. In the case of finitely many alternatives, we provide a characterization of monotonic preferences which relaxes the submodularity axiom of Kreps (1979). We characterize the minimal state space needed for our representation, and we show that the second-period choice behavior of our decision maker differs from the one implied by the costly contemplation model of Ergin (2003).
\end{abstract}

(c) 2018 Elsevier B.V. All rights reserved.

\section{Introduction}

This paper analyzes preferences over menus of options. A preference over menus is monotonic when every menu is at least as good as any of its subsets. Theorem 1 shows that every utility representation for a monotonic preference is equal to the minmax value of a decision maker whose payoff depends on the option chosen from the menu and on the realization of a subjective state. This representation suggests a decision maker who faces uncertainty about her own future tastes and who exhibits an extreme form of pessimism with respect to this uncertainty.

We explore the consequences of Theorem 1 in the special case of a finite set of choice objects. This is the setting of the preference for flexibility model of Kreps (1979). Our pessimism utility representation result generalizes Kreps' model by relaxing the submodularity axiom. We provide a characterization of the minimal number of states necessary to write our representation.

The costly contemplation model of Ergin (2003) also generalizes Kreps' model in the finite setting. While the patterns of first-period choice among menus in our model are equivalent to those of Ergin's model, we show that costly contemplation and pessimism produce different predictions for second-period choice from menus.

\section{Preference for flexibility}

Let $Z$ be an arbitrary set of choice objects and let $X \subset 2^{Z} \backslash\{\varnothing\}$ be a collection of non-empty menus containing $Z$. A preference over menus is given by a binary relation $\succcurlyeq \subset X \times X$. A preference over menus is monotonic if $x \supset x^{\prime}$ implies $x \succcurlyeq x^{\prime}$. A function $U: X \rightarrow \mathbb{R}$ is a utility representation for $\succcurlyeq$ whenever for all $x, x^{\prime} \in X$ we have $x \succcurlyeq x^{\prime}$ if and only if $U(x) \geq U\left(x^{\prime}\right)$. Our

\footnotetext{
* Corresponding author.

E-mail addresses: leandro.gorno@fgv.br (L. Gorno), pnatenzon@wustl.edu (P. Natenzon).
} 
main result is that any utility representation for a monotonic preference is equal to the minmax value of a decision maker whose payoff depends on the option chosen from the menu and on the realization of a subjective state.

Theorem 1. Every representation $U$ for a monotonic preference $\succcurlyeq$ can be written as

$$
U(x)=\min _{s \in S} \max _{z \in X} u(z, s)
$$

where $S$ is a set of subjective states and $u: Z \times S \rightarrow \mathbb{R}$ is a state-dependent utility.

Proof. Since $U$ represents $\succcurlyeq$ it must be bounded above, attaining its maximum at $Z$, the largest menu. Assume without loss of generality that $U(Z)=0$.

Take $S=X$ and for each menu $s \in S$ let $\mathbb{I}_{S}: Z \rightarrow \mathbb{R}$ be the indicator function defined by $\mathbb{I}_{S}(z)=1$ if $z \in S$ and $\mathbb{I}_{S}(z)=0$ otherwise. Define $u: Z \times S \rightarrow \mathbb{R}$ by $u(z, s)=U(s) \mathbb{I}_{S}(z)$ for each $z \in Z$ and $s \in S$.

Note that for any $s \in S$ we have $\max _{z \in x} u(z, s)$ equal to $U(s)$ whenever $x \subset s$ and equal to zero otherwise. Also whenever $s \supset x$ we have $s \succcurlyeq x$ and therefore $U(s) \geq U(x)$. Moreover, for each $x \in X$ the set $\left\{\max _{z \in X} u(z, s): s \in S\right\}$ attains its minimum at $s=x$. Hence the right-hand side of $(1)$ is a well-defined function of $x$ and for all $x \in X$ we have

$$
\begin{aligned}
\min _{s \in S} \max _{z \in X} u(z, s) & =\min _{s \supset X} \max _{z \in X} u(z, s) \\
& =\min _{s \supset X} U(s) \\
& =U(x) .
\end{aligned}
$$

The representation (1) has the following interpretation. The decision maker chooses a menu today as if she were unsure about what her ranking of the alternatives in $Z$ will be tomorrow. She foresees the possibilities represented by the state space $S$. Each state $s \in S$ yields a utility index $z \mapsto u(z, s)$ representing a possible ranking over the objects in $Z$. She is extremely pessimistic when evaluating which subjective state will occur, assessing each menu according to the worst-case scenario.

A parallel can be drawn between representation (1) and the multiple prior model of Gilboa and Schmeidler (1989). To see this formally, identify the choice of a menu $x \in X$ with an act $f_{X}: S \rightarrow Z \times S$ that delivers a payoff $f_{X}(s)=\left(\arg \max _{z \in X} u(z, s), s\right)$ which depends on the realization of the uncertain state $s \in S$. The decision maker exhibits an extreme form of ambiguity aversion and has a set of priors equal to the entire simplex $\Delta(S)$. She evaluates each menu $x \in X$ (represented by the act $f_{x}$ ) according to

$$
\begin{aligned}
U(x) & =\min _{s \in S} \max _{z \in X} u(z, s) \\
& =\min _{\mu \in \Delta(S)} \sum_{s \in S} \mu(s) \max _{z \in X} u(z, s) \\
& =\min _{\mu \in \Delta(S)} \int_{S} u \circ f_{X} d \mu
\end{aligned}
$$

which is the well-known functional form in the multiple prior model.

However, our model differs from Gilboa and Schmeidler's in two fundamental ways. First, the choice objects in the multiple prior model are acts, which map a set of states to a set of consequences, while our choice objects are menus of options. Second, in the multiple prior model the set of states is a primitive. In contrast, the state space in Theorem 1 is subjective, unobserved and obtained as part of the representation.

Remark 1. Theorem 1 is silent on the existence of a utility representation. These conditions are well-known. In particular, it follows immediately from Theorem 1 above and Theorem 3.1 in Fishburn (1970), that a preference relation $\succcurlyeq \subset X \times X$ admits a utility representation $U: X \rightarrow \mathbb{R}$ given by (1) if and only if it is complete, transitive, monotonic, and has an order-dense countable subset.

\section{Finite environments}

In this section, we explore the consequences of Theorem 1 when the set $Z$ of choice objects is finite. This is the setting of the preference for flexibility model of Kreps (1979) and the costly contemplation model of Ergin (2003). Let $X=2^{Z} \backslash\{\varnothing\}$ be the collection of all non-empty menus of objects from $Z$. Kreps (1979) imposes the following axioms on the preference $\succcurlyeq \subset \times X$ :

Axiom (A1). Weak order: $\succcurlyeq$ is complete and transitive.

Axiom (A2). Monotonicity: $x \supset x^{\prime}$ implies $x \succcurlyeq x^{\prime}$.

Axiom (A3). Submodularity: $x \sim x \cup x^{\prime}$ implies $x \cup x^{\prime \prime} \sim x \cup x^{\prime} \cup x^{\prime \prime}$ for all $x^{\prime \prime} \in X$.

A1 is standard and allows a utility representation in this finite setting. A2 means the decision maker never values commitment, yet allows preference for flexibility. A3 says that if the decision maker does not value the flexibility provided by 
adding $x^{\prime}$ to $x$, then she must also not value adding the same $x^{\prime}$ to the larger $x \cup x^{\prime \prime}$. Kreps (1979) proved that $\succcurlyeq$ satisfies A1-A3 if and only if it admits the following representation:

Definition. $U: X \rightarrow \mathbb{R}$ is an additive utility if there exist parameters $(S, u, \mu)$ where $S$ is a finite set of subjective states, $u: Z \times S \rightarrow \mathbb{R}$ is a state dependent utility index, and $\mu$ is a probability on $S$ with full support such that, for all $x \in X$,

$$
U(x)=\sum_{s \in S} \mu(s) \max _{z \in X} u(z, s) .
$$

The content of Kreps' representation is ordinal: the probability measure $\mu$ cannot be identified in the finite setting, and the expectation operator in (2) can be replaced with other strictly increasing aggregators of the vector of second-period indirect utilities. 1

Theorem 1 suggests a model which uses the min operator in place of the expectation operator used in Kreps' additive representation.

Definition. A utility function $V: X \rightarrow \mathbb{R}$ is a pessimism utility if there exist parameters $(S, u)$ where $S$ is a finite set, $u$ : $Z \times S \rightarrow \mathbb{R}$, and for each $x \in X$

$$
V(x)=\min _{s \in S} \max _{z \in X} u(z, s) .
$$

Both our decision maker and Kreps' foresee a finite set $S$ of subjective states and a state-dependent utility $u: Z \times S \rightarrow \mathbb{R}$ which describes their preferences in each state. However, our decision maker evaluates each menu according to the worstcase scenario for the realization of the state. The following characterization result shows that our model generalizes Kreps' additive model by relaxing the submodularity axiom A3:

Proposition 2. $\succcurlyeq$ has a pessimism utility representation iff it satisfies A1 and A2.

Proof. Necessity is straightforward. For sufficiency, note that since $Z$ is finite, $X$ is finite, and under A1 $\succcurlyeq$ admits a utility representation $V: X \rightarrow \mathbb{R}$. Apply Theorem 1 to find parameters $(S, u)$ such that (3) holds. It is clear from the proof of Theorem 1 that $S$ can be taken to be finite in this case.

Example 1. Consider the decision problem of an individual who needs to buy pasta to cook for dinner next Sunday (cf. the car dealership choice example in Ergin (2003)). The pasta alternatives are manicotti, linguine and tagliatelle, and accordingly we write $Z=\{m, \ell, t\}$. We observe her preferences over menus, given by

$$
\{m, \ell, t\} \succ\{\ell, t\} \succ\{m, t\} \sim\{t\} \succ\{m, \ell\} \sim\{\ell\} \succ\{m\}
$$

which satisfy A1 and A2 but not A3. For these particular preferences, an additive utility representation as in (2) is not possible, but it is easy to find a pessimism utility representation. Define $S=\left\{s_{1}, s_{2}, s_{3}\right\}$ and let $u: Z \times S \rightarrow \mathbb{R}$ be summarized by

\begin{tabular}{l|rrr} 
& $s_{1}$ & $s_{2}$ & $s_{3}$ \\
\hline$u(m, \cdot)$ & 4 & 1 & 0 \\
$u(\ell, \cdot)$ & 3 & 5 & 1 \\
$u(t, \cdot)$ & 2 & 2 & 5
\end{tabular}

It is easy to check that, with these choices of $(S, u)$, the function $V: X \rightarrow \mathbb{R}$ defined in (3) represents $\succcurlyeq$. $\diamond$

The decision maker in Example 1 values flexibility, in the sense that having more varieties of pasta available never hurts. The pessimism utility with parameters $(S, u)$ provided in Example 1 suggests a decision maker who anticipates three possible subjective states when the time comes to choose a type of pasta to cook for dinner on Sunday. Each menu is evaluated according to the worst-case scenario for the realization of the state, which allows for violations of the submodularity axiom (A3). For example, adding the manicotti pasta to the menu that only has linguine leaves the decision maker indifferent, since in both cases the worst-case scenario is the realization of state $s_{3}$ and the maximum attainable utility in both cases is 1. But adding the same manicotti pasta to a menu that already contains linguine and tagliatelle leaves the decision maker strictly better off: the worst-case scenario when the options are $\{\ell, t\}$ is the realization of state $s_{1}$, where the maximum attainable payoff is 3 , while having the options $\{m, \ell, t\}$ guarantees a payoff of 4 .

1 Dekel et al. (2001, 2007) obtain uniqueness results by enriching the set choice of objects with a lottery structure and imposing a normalization on the subjective state space. Epstein et al. (2007) consider ambiguity in this enriched setting and obtain uniqueness results for a set of beliefs over the subjective state space. Sadowski (2013) shows beliefs can be uniquely identified in a model with an exogenous state space of publicly observable information when choice is assumed to be sufficiently dependent on information. Other recent advances in the identification of beliefs on a subjective state space include Ergin and Sarver (2010), Chatterjee and Krishna (2011), Ahn and Sarver (2013), and Schenone (2016). 


\subsection{Minimal subjective state space}

The size of the subjective state space can be thought of as providing a measure of the degree of uncertainty faced by the decision maker when choosing a menu in the first period. ${ }^{2}$ A function $U: X \rightarrow \mathbb{R}$ is an indirect utility whenever $U(x)=\max _{z \in X} U(\{z\})$ for all $x$. It is easy to verify that $U$ is an indirect utility if and only if it satisfies (2) and (3) with a single subjective state. In contrast, our characterization below implies that any parameterization $(S, u)$ of a pessimism utility representing the preferences in Example 1 requires at least three states.

In order to state our minimality result, we need to introduce some additional definitions. First, let $\mathcal{F}(\succcurlyeq):=$ $\{x \in X: y \sim x \subset y$ implies $y=x\}$ be the collection of menus which are not indifferent to any of its supersets. For example, in the case of the preference relation $\succcurlyeq$ in Example $1, \mathcal{F}(\succcurlyeq)$ consists of the sets $\{m, \ell, t\},\{\ell, t\},\{m, t\},\{m, \ell\}$, and $\{m\}$. A chain in $\mathcal{F}(\succcurlyeq)$ is any subset of $\mathcal{F}(\succcurlyeq)$ totally ordered by set inclusion. For instance, $\{\{m, \ell, t\},\{m, t\}$, $\{m\}\}$ is a chain in $\mathcal{F}(\succcurlyeq)$ in Example 1 since $\{m, \ell, t\} \supset\{m, t\} \supset\{m\}$. We define the chain covering number of $\succcurlyeq$, denoted by $N(\succcurlyeq)$, as the smallest number $n \in \mathbb{N}$ such that there is a cover of $\mathcal{F}(\succcurlyeq)$ consisting of $n$ chains in $\mathcal{F}(\succcurlyeq){ }^{3}$ In Example 1 we have $N(\succcurlyeq)=3$ since $\mathcal{F}(\succcurlyeq)$ can be covered by three chains in $\mathcal{F}(\succcurlyeq)$ (e.g. $\{\{m, \ell, t\},\{m, t\},\{m\}\},\{\{m, \ell, t\},\{m, \ell\}\}$, and $\{\{\ell, t\}\})$ but $\mathcal{F}(\succcurlyeq)$ cannot be covered by any two chains in $\mathcal{F}(\succcurlyeq)$. We prove this minimality result in the Appendix:

Theorem 3. If the pessimism utility $U$ represents $\succcurlyeq$, there exist parameters $(S, u)$ for $U$ with $|S|=N(\succcurlyeq)$. Moreover, any parameters $\left(S^{\prime}, u^{\prime}\right)$ for $U$ satisfy $\left|S^{\prime}\right| \geq N(\succcurlyeq)$.

Theorem 3 implies that the minimal number of subjective states necessary to parameterize a pessimism utility representation is solely determined by the ordinal information contained in $\succcurlyeq$ : it does not depend on the particular utility representation chosen. ${ }^{4}$ Example 2 in Section 3.3 shows that an analogous characterization of minimality is not possible for the models of Kreps (1979) and Ergin (2003).

\subsection{Pessimism and additivity}

When the preference relation $\succcurlyeq$ satisfies axioms A1, A2, and A3 it admits both an additive utility representation and a pessimism utility representation. ${ }^{5}$ In fact, it is possible to find a pair of representations as in (2) and (3) that share the same finite subjective state space $S$ and state dependent utility function $u$. We leave the proof of this result to the Appendix:

Proposition 4. $\succcurlyeq$ satisfies A1, A2, and A3 iff there exist a finite state space $S$ and a state dependent utility $u$ such that $\succcurlyeq$ admits an additive utility representation with parameters $(S, u, \mu)$ and a pessimism utility representation with parameters $(S, u)$.

The main takeaway is that the submodularity axiom A3 corresponds in the representation to a property of the aggregator of second-period indirect utilities. Say that a function $\varphi: \mathbb{R}^{n} \rightarrow \mathbb{R}$ is increasing if $a_{i}>b_{i}$ for all $i=1, \ldots, n$ implies $\varphi(a)>\varphi(b)$. It is strictly increasing if $a_{i} \geq b_{i}$ for all $i=1, \ldots, n$ and $a \neq b$ implies $\varphi(a)>\varphi(b)$. If $w: X \rightarrow \mathbb{R}^{S}$ is defined by

$$
(w(x))(s)=\max _{z \in X} u(z, s),
$$

and $\varphi: \mathbb{R}^{S} \rightarrow \mathbb{R}$ is an aggregator of second-period utilities, then $U=\varphi \circ w$ if $\varphi$ is the strictly increasing expectation aggregator in (2), while $V=\varphi \circ w$ when $\varphi$ is the increasing min aggregator in (3). As Theorem 1' in Kreps (1979) shows, a preference satisfies A3 (in addition to A1 and A2) if and only if we can find a strictly increasing aggregator $\varphi$ such that $\varphi \circ w$ represents $\succcurlyeq$.

\subsection{Pessimism versus costly contemplation}

Our decision maker is pessimistic: she evaluates each menu of options using the worst-case scenario for the realization of the subjective state. Our representation generalizes Kreps' additive model by relaxing the submodularity axiom A3. Ergin (2003) also generalizes Kreps with a model of costly contemplation:

Definition. $U: X \rightarrow \mathbb{R}$ is a contemplation utility if there exist parameters $(S, u, \mu, \mathcal{I}, c)$ where $S$ is a finite set, $u: Z \times S \rightarrow \mathbb{R}$, $\mu$ is a probability on $S$ with full support, $\mathcal{I}$ is a set of partitions of $S$ containing the trivial partition $\{S\}$, and $c: \mathcal{I} \rightarrow[0, \infty)$ with $c(\{S\})=0$ such that, for all $x \in X$,

$$
U(x)=\max _{\pi \in \mathcal{I}}\left[\sum_{E \in \pi} \max _{z \in X} \sum_{s \in E} \mu(s) u(z, s)-c(\pi)\right] .
$$

\footnotetext{
2 Kreps (1979) suggests using chains of menus to economize on the number of states needed for his representation. We build on this idea to obtain the minimal number of states for our model. Gorno (2016) characterizes the minimal state space for a given additive utility function, with or without monotonicity. Kopylov (2018) uses combinatorial techniques to study minimal state spaces in general finite environments.

3 By Dilworth's theorem, $N(\succcurlyeq)$ is also equal to the size of the largest antichain of $\mathcal{F}(\succcurlyeq)$ (see Chapter 2 in Harzheim (2005) for details).

4 The invariance of the representation in Theorem 1 can be obtained directly by noting that, if $(S, u)$ are parameters for a pessimism utility representation of $\succcurlyeq$ and $S^{\prime}$ is any set with the same cardinality as $S$, then every utility representation for $\succcurlyeq$ is a pessimism utility with state space $S^{\prime}$.

5 Analogously, in the domain of preferences over acts, Dillenberger et al. (2017) show the existence of pessimistic and optimistic representations that satisfy all of the axioms of subjective expected utility in Savage (1954).
} 
Ergin (2003) shows that any utility representation for a monotonic preference is a contemplation utility. The formula in (4) generalizes Kreps' additive utility representation by adding two parameters: the set $\mathcal{I}$ of contemplation strategies, and the contemplation cost function $c$. The decision maker's valuation of a menu reflects an optimal trade-off between acquiring more refined information about the state and keeping contemplation costs down. For example, consider how this model rationalizes the preferences in Example 1. Following Ergin (2003), let the state space be $S=\left\{s_{1}, s_{2}, s_{3}\right\}$, let the state dependent utility be given by

\begin{tabular}{l|rrr} 
& $s_{1}$ & $s_{2}$ & $s_{3}$ \\
\hline$u(m, \cdot)$ & -5 & 0 & 5 \\
$u(\ell, \cdot)$ & -2 & 1 & 4 \\
$u(t, \cdot)$ & 0 & 0 & 0
\end{tabular}

and let the decision maker's prior beliefs be given by $\mu\left(s_{1}\right)=0.6, \mu\left(s_{2}\right)=0.3$, and $\mu\left(s_{3}\right)=0.1$. The decision maker has two contemplation strategies available in $\mathcal{I}$ : the first contemplation strategy is to not contemplate at all. The state of knowledge in this case is described by the trivial partition of the subjective state space $\{S\}$ and carries a contemplation cost $c(\{S\})=0$. The second contemplation strategy perfectly reveals the state. It corresponds to the partition $\left\{\left\{s_{1}\right\},\left\{s_{2}\right\},\left\{s_{3}\right\}\right\}$ and carries a contemplation cost $c\left(\left\{\left\{s_{1}\right\},\left\{s_{2}\right\},\left\{s_{3}\right\}\right\}\right)=0.6$.

This decision maker dislikes thinking about what kind of pasta is the best option for dinner on Sunday. The presence of costly contemplation allows the model to violate the submodularity axiom A3. For instance, adding the manicotti pasta to the menu that contains the single linguine option is not enough of an incentive to engage in costly contemplation. Contemplating carries a utility cost of 0.6 ; the benefit of contemplating is being able to cook manicotti instead of linguine whenever the unlikely subjective state $s_{3}$ is realized, which only brings an expected utility gain of 0.1 . We have $\{m, \ell\} \sim\{\ell\}$ because in both menus the decision maker optimally avoids contemplation and cooks the linguine.

In contrast, adding the same manicotti pasta option to the menu that contains linguine and tagliatelle leaves the decision maker strictly better off. The presence of linguine and tagliatelle makes it worth it to contemplate and fully reveal the subjective state. Contemplating allows the decision maker to cook the linguine whenever states $s_{2}$ and $s_{3}$ occur. This in turn brings an expected utility gain of 0.7 , which more than compensates the utility cost of contemplation. Once contemplation is optimal, adding the manicotti option brings an additional expected utility gain while incurring no additional contemplation cost. Hence $\{m, \ell, t\} \succ\{\ell, t\}$, violating A3.

Any preference $\succcurlyeq$ satisfying axioms $A 1$ and $A 2$ can be represented by some pessimism utility with parameters $(S$, $u)$, and also by some contemplation utility with parameters $\left(S^{\prime}, u^{\prime}, \mu, \mathcal{I}, c\right)$. The minimal number of states needed for the pessimism utility representation is characterized in Theorem 3. This minimality result has no counterpart in the contemplation model. The example below shows that two contemplation utilities that represent the same preference may require different numbers of subjective states, and that the same is true for additive utility representations.

Example 2. Let $Z=\left\{z_{1}, z_{2}, z_{3}\right\}$ and let $U_{\alpha}: X \rightarrow \mathbb{R}$ be given by

$$
\begin{array}{r}
U_{\alpha}\left(\left\{z_{1}, z_{2}, z_{3}\right\}\right)=U_{\alpha}\left(\left\{z_{1}, z_{2}\right\}\right)=1, \\
U_{\alpha}\left(\left\{z_{1}, z_{3}\right\}\right)=U_{\alpha}\left(\left\{z_{2}, z_{3}\right\}\right)=\alpha, \\
U_{\alpha}\left(\left\{z_{1}\right\}\right)=U_{\alpha}\left(\left\{z_{2}\right\}\right)=U_{\alpha}\left(\left\{z_{3}\right\}\right)=0,
\end{array}
$$

where $1>\alpha>0$. The preferences represented by $U_{\alpha}$ satisfy the submodularity axiom A3, in addition to A1 and A2. $U_{\alpha}$ is a contemplation utility for all $0<\alpha<1$. When $\alpha \leq 1 / 2$, there is a parameterization for the contemplation utility $U_{\alpha}$ using two states. Let $S=\left\{s_{1}, s_{2}\right\}$, let $u: Z \times S \rightarrow \mathbb{R}$ be given by

\begin{tabular}{c|cc} 
& $s_{1}$ & $s_{2}$ \\
\hline$u\left(z_{1}, \cdot\right)$ & $4-4 \alpha$ & $4 \alpha-4$ \\
$u\left(z_{2}, \cdot\right)$ & 0 & 0 \\
$u\left(z_{3}, \cdot\right)$ & $2-2 \alpha$ & $2 \alpha-2$
\end{tabular}

and let prior beliefs be given by $\mu\left(s_{1}\right)=\mu\left(s_{2}\right)=1 / 2$. Let the set of contemplation strategies be $\mathcal{I}=\left\{\{S\}\right.$, $\left.\left\{\left\{s_{1}\right\},\left\{s_{2}\right\}\right\}\right\}$, with contemplation costs given by $c(\{S\})=0$ and $c\left(\left\{\left\{s_{1}\right\},\left\{s_{2}\right\}\right\}\right)=1-2 \alpha \geq 0$. Direct calculation shows that $U_{\alpha}$ is a contemplation utility with parameters $(S, u, \mu, \mathcal{I}, c)$. When $\alpha>1 / 2$, however, any contemplation utility parameterization of $U_{\alpha}$ requires at least three states (we leave the proof to the reader).

Now consider additive utility representations. A characterization result of Nehring (1999) implies that $U_{\alpha}$ is an additive utility if and only if it satisfies the inequalities

$$
\begin{aligned}
& U_{\alpha}\left(\left\{z_{1}, z_{2}, z_{3}\right\}\right)-U_{\alpha}\left(\left\{z_{1}, z_{2}\right\}\right)-U_{\alpha}\left(\left\{z_{1}, z_{3}\right\}\right)+U_{\alpha}\left(\left\{z_{1}\right\}\right) \leq 0 \\
& U_{\alpha}\left(\left\{z_{1}, z_{2}, z_{3}\right\}\right)-U_{\alpha}\left(\left\{z_{1}, z_{2}\right\}\right)-U_{\alpha}\left(\left\{z_{2}, z_{3}\right\}\right)+U_{\alpha}\left(\left\{z_{2}\right\}\right) \leq 0 \\
& U_{\alpha}\left(\left\{z_{1}, z_{2}, z_{3}\right\}\right)-U_{\alpha}\left(\left\{z_{1}, z_{3}\right\}\right)-U_{\alpha}\left(\left\{z_{2}, z_{3}\right\}\right)+U_{\alpha}\left(\left\{z_{3}\right\}\right) \leq 0
\end{aligned}
$$

and direct calculation shows the inequalities hold if and only if $\alpha \geq 1 / 2$. Hence, $U_{\alpha}$ is not an additive utility for $\alpha<1 / 2$. For the special case $\alpha=1 / 2$ the cost function $c$ constructed above is constant and equal to zero, and therefore $U_{1 / 2}$ is an additive utility with two states. For $\alpha>1 / 2$, contemplation utility parameters for $U_{\alpha}$ require at least three states. Hence, the same holds for additive utility parameterizations. $\diamond$ 
While the pessimism and contemplation models generate the same set of choice behaviors in the first period, we show, below, that these models generate different predictions for second-period behavior.

\subsection{Second-period behavior}

In this section, we study the second-period behavior implied by a pessimism utility representation and show how it differs from the behavior implied by the contemplation model. The function $\lambda: X \times X \rightarrow[0,1]$ is a second-period choice rule if, for every menu $x$,

$$
\sum_{x^{\prime} \subset x} \lambda\left(x^{\prime}, x\right)=1,
$$

and, moreover, $\lambda\left(x^{\prime}, x\right)=0$ whenever $x^{\prime} \not \subset x$. The preference $\succcurlyeq$ describes how the decision maker chooses among menus in the first period, while the choice rule $\lambda$ describes choices from menus in the second period. A second-period choice rule $\lambda$ is resolute if it never expresses indifference, that is, $\lambda\left(x^{\prime}, x\right)>0$ implies $x^{\prime}=\{z\}$ for some $z \in x$. A second-period choice rule $\lambda$ is deterministic if $\lambda\left(x^{\prime}, x\right)$ is always equal to zero or one.

We use second-period choice rules to formalize our decision maker's anticipation (in the first period) of her choice behavior when the time comes to pick an option from the chosen menu. A second-period choice rule $\lambda$ is consistent with pessimism utility parameters $(S, u)$ if $\lambda\left(x^{\prime}, x\right)>0$ implies there exists a state $s^{\prime} \in S$ with $u\left(z^{\prime}, s^{\prime}\right)=\min _{s \in S} \max _{z \in x} u(z, s)>u\left(z^{\prime \prime}, s^{\prime}\right)$ for every $z^{\prime} \in X^{\prime}$ and every $z^{\prime \prime} \in X \backslash x^{\prime}$. For example, the unique $\lambda$ consistent with the parameters $(S, u)$ given in Example 1 is

$$
\lambda(\{m\},\{m, \ell, t\})=\lambda(\{\ell\},\{\ell, t\})=\lambda(\{t\},\{m, t\})=\lambda(\{\ell\},\{m, \ell\})=1,
$$

which is both resolute and deterministic.

The decision maker described by the pessimism utility parameters in Example 1 anticipates the worst case scenario $s_{1}$ when the menu is $\{m, \ell, t\}$ and the worst case scenario $s_{2}$ when the menu is $\{\ell, t\}$. This decision maker can be described as someone who anticipates a correlation between the chosen menu and the subjective state realization. In contrast, the decision maker described by the contemplation model anticipates the subjective state realization to be independent of firstperiod menu choice. We now show this leads to differences in second-period behavior between the two representations.

Consider the second-period behavior anticipated by the decision maker in the costly contemplation model. A secondperiod choice rule $\lambda$ is consistent with contemplation utility parameters $(S, u, \mu, \mathcal{I}, c)$, when, for each menu $x$, there exists an optimal contemplation strategy $\pi \in \mathcal{I}$ under which $\lambda\left(x^{\prime}, x\right)$ is equal to the probability given by $\mu$ to the event in $\pi$ in which the set of choice options in $x$ that tie for maximum expected utility is $x^{\prime}$. For instance, the unique $\lambda$ consistent with the contemplation parameters $(S, u, \mu, \mathcal{I}, c)$ used in Section 3.3 to rationalize the preferences in Example 1 is

$$
\begin{aligned}
\lambda(\{m\},\{m, \ell, t\}) & =1 / 10, \\
& \lambda(\{\ell\},\{m, \ell, t\})=3 / 10, \\
& \lambda(\{t\},\{m, \ell, t\})=6 / 10, \\
\lambda(\{t\},\{m, t\})=\lambda(\{t\},\{\ell, t\})=\lambda(\{\ell\},\{m, \ell\}) & =1,
\end{aligned}
$$

which is resolute but not deterministic. In fact, our next result implies that there does not exist a deterministic secondperiod choice rule consistent with any parameters of any contemplation utility that represent the preferences in Example 1 .

Proposition 5. If a second-period choice rule $\lambda$ is consistent with contemplation utility parameters $(S, u, \mu, \mathcal{I}, c)$ and deterministic, then the contemplation utility parameterized by $(S, u, \mu, \mathcal{I}, c)$ is an indirect utility.

Proof. An option $z$ is essential in $x$ if $x=\{z\}$ or if $x>x \backslash\{z\}$. Ergin (2003) shows that if $z$ is essential in $x$, then in any optimal contemplation strategy for $x$, there exists an event with strictly positive probability in which $z$ is the unique expected utility maximizer among the options in $x$. In other words, if $z$ is essential in $x$, we must have $\lambda(\{z\}, x)>0$ for every second-period choice rule $\lambda$ that is consistent with any contemplation utility parameters that represent $\succcurlyeq$. If one such $\lambda$ is deterministic, then at most one element $z$ can be essential in each menu $x$. And in this case Ergin (2003) $\operatorname{shows}$ that $U(x)=\max _{z \in x} U(\{x\})$ for all $x$, that is, $U$ is an indirect utility.

Proposition 5 implies that, in Example 1, the second-period choice rule $\lambda$ given by equation (6) is consistent with the pessimism model but not consistent with the contemplation model. Conversely, Corollary 7 and Example 3, below, show that there are instances in which a second-period choice rule $\lambda$ is consistent with costly contemplation but not with pessimism.

Proposition 6. Let $(S, u)$ be parameters of a pessimism utility $U$ and consider three menus $x, x^{\prime}, x^{\prime \prime} \in X$ such that $x \cap x^{\prime}=\varnothing$ and $x^{\prime \prime} \subset x^{\prime}$. Then, $U\left(x \cup x^{\prime}\right)=U(x)$ implies $\lambda\left(x^{\prime \prime}, x \cup x^{\prime}\right)=0$ for any second-period choice rule $\lambda$ consistent with $(S, u)$.

Proof. Assume $\lambda$ is consistent with $(S, u)$ and $\lambda\left(x^{\prime \prime}, x \cup x^{\prime}\right)>0$. Then there exists a state $s^{\prime} \in S$ such that for all $z \in \mathcal{X}$ and $z^{\prime} \in \mathcal{X}^{\prime \prime}$ we have $U\left(x \cup x^{\prime}\right)=u\left(z^{\prime}, s^{\prime}\right)>u\left(z, s^{\prime}\right)$. But then $U(x)=\min _{s \in S} \max _{z \in X} u(z, s) \leq \max _{z \in X} u\left(z, s^{\prime}\right)<u\left(z^{\prime}, s^{\prime}\right)=U\left(x \cup x^{\prime}\right)$.

Corollary 7. If $U$ is a pessimism utility and $U(x)=U(\{z\})$ for all $z \in x$, then $\lambda(x, x)=1$ for every $\lambda$ consistent with some parameters of $U$. 
Proof. By Proposition $6, \lambda\left(x^{\prime}, x\right)=0$ whenever $x^{\prime} \neq x$. Hence $\lambda(x, x)=1$.

Example 3. Let there be three options $Z=\left\{z_{1}, z_{2}, z_{3}\right\}$ and consider the preferences

$$
\left\{z_{1}, z_{2}, z_{3}\right\} \succ\left\{z_{1}, z_{2}\right\} \sim\left\{z_{1}, z_{3}\right\} \sim\left\{z_{2}, z_{3}\right\} \sim\left\{z_{1}\right\} \sim\left\{z_{2}\right\} \sim\left\{z_{3}\right\}
$$

which satisfy weak order (A1) and monotonicity (A2) but fail submodularity (A3). Consider the following costly contemplation utility parameters $(S, u, \mu, \mathcal{I}, c)$. Let there be three states $S=\left\{s_{1}, s_{2}, s_{3}\right\}$ and let the state dependent utility given by

\begin{tabular}{l|rrr} 
& $s_{1}$ & $s_{2}$ & $s_{3}$ \\
\hline$u\left(z_{1}, \cdot\right)$ & 1 & 3 & 2 \\
$u\left(z_{2}, \cdot\right)$ & 2 & 1 & 3 \\
$u\left(z_{3}, \cdot\right)$ & 3 & 2 & 1
\end{tabular}

Each state is equally likely in the prior $\mu\left(s_{1}\right)=\mu\left(s_{2}\right)=\mu\left(s_{3}\right)=1 / 3$. The set of contemplation strategies $\mathcal{I}$ includes all possible partitions of $S$. The cost of no contemplation is zero $c(\{S\})=0$, and any other contemplation strategy has cost $2 / 3$. It is straightforward to verify that the costly contemplation utility with these parameters represents the preferences above. A second-period choice rule $\lambda$ consistent with $(S, u, \mu, \mathcal{I}, c)$ is given by:

$$
\begin{array}{r}
\lambda\left(\left\{z_{1}\right\},\left\{z_{1}, z_{2}, z_{3}\right\}\right)=\lambda\left(\left\{z_{2}\right\},\left\{z_{1}, z_{2}, z_{3}\right\}\right)=\lambda\left(\left\{z_{3}\right\},\left\{z_{1}, z_{2}, z_{3}\right\}\right)=1 / 3 \\
\lambda\left(\left\{z_{1}\right\},\left\{z_{1}, z_{2}\right\}\right)=\lambda\left(\left\{z_{2}\right\},\left\{z_{2}, z_{3}\right\}\right)=\lambda\left(\left\{z_{3}\right\},\left\{z_{1}, z_{3}\right\}\right)=1 / 3 \\
\lambda\left(\left\{z_{2}\right\},\left\{z_{1}, z_{2}\right\}\right)=\lambda\left(\left\{z_{3}\right\},\left\{z_{2}, z_{3}\right\}\right)=\lambda\left(\left\{z_{1}\right\},\left\{z_{1}, z_{3}\right\}\right)=2 / 3
\end{array}
$$

Since this $\lambda$ is resolute, Corollary 7 implies $\lambda$ is not consistent with the parameters of any pessimism utility representation of $\succcurlyeq . \diamond$

\section{Acknowledgment}

We are grateful to the co-editor, Daniela Puzzello, an associate editor, and the referees for very helpful comments. Paulo Natenzon gratefully acknowledges financial support from the Weidenbaum Center on the Economy, Government, and Public Policy at Washington University in Saint Louis.

\section{Appendix. Omitted proofs}

\section{Proof of Theorem 3}

The argument consists of three lemmas. Lemma 8 constructs a representation for each collection of chains covering $\mathcal{F}(\succcurlyeq)$. Lemma 9 shows the construction in Lemma 8 is efficient when only one state is needed. Lemma 10 establishes minimality.

Lemma 8. Let $U$ be a pessimism utility representation of $\succcurlyeq$ and let $S$ be a collection of chains in $\mathcal{F}(\succcurlyeq)$ covering $\mathcal{F}(\succcurlyeq)$. Then, there exists $u: Z \times S \rightarrow \mathbb{R}$ such that $(S, u)$ are parameters for $U$.

Proof. Define $u: Z \times S \rightarrow \mathbb{R}$ by

$$
u(z, s):=\min \left\{\min \left\{U\left(x^{\prime}\right): z \in x^{\prime} \in s\right\}, U(Z)+1\right\}
$$

for each $z \in Z$ and $s \in S$. The outer min ensures that $u(z, s)$ is finite for all $z \in Z$ and $s \in S$.

Fix a menu $x \in X$ and a state $s \in S$. If $x \subset x^{\prime}$ for some $x^{\prime} \in S$, then $\max _{z \in X} u(z, s)=\min \left\{U\left(x^{\prime}\right): x \subset x^{\prime} \in s\right\}$. Otherwise, we have $\max _{z \in X} u(z, s)=U(Z)+1$. Combining both cases, we obtain

$$
\max _{z \in X} u(z, s)=\min \left\{\min \left\{U\left(x^{\prime}\right): x \subset x^{\prime} \in s\right\}, U(Z)+1\right\} .
$$

Since $S$ covers $\mathcal{F}(\succcurlyeq)$, for every $x \in X$, we can find $s \in S$ and $x^{\prime} \in S$ such that $x \subset x^{\prime}$ and $U(x)=U\left(x^{\prime}\right)$. It follows that

$$
\begin{aligned}
\min _{s \in S} \max _{z \in X} u(z, s) & =\min \left\{\min \left\{U\left(x^{\prime}\right): x \subset x^{\prime} \in S \in S\right\}, U(Z)+1\right\} \\
& =\min \left\{U\left(x^{\prime}\right): x \subset x^{\prime} \in S \in S\right\} \\
& =U(x)
\end{aligned}
$$

as desired.

Lemma 9. If $\succcurlyeq$ can be represented by an indirect utility, then $\mathcal{F}(\succcurlyeq)$ is a chain. 
Proof. If $U$ is an indirect utility that represents $\succcurlyeq$, there is $u: Z \rightarrow \mathbb{R}$ such that $U(x)=\max _{z \in X} u(z)$ for all $x \in X$. Then, we can explicitly obtain

$$
\mathcal{F}(\succcurlyeq)=\{\{z \in Z: u(z) \leq r\}: r \in u(Z)\},
$$

which is clearly a chain.

Considering Lemma 8, we say that the parameters $(S, u)$ of a pessimism utility representation of $\succcurlyeq$ are canonical if $S$ is a collection of chains in $\mathcal{F}(\succcurlyeq)$ covering $\mathcal{F}(\succcurlyeq)$.

Lemma 10. If $(S, u)$ are parameters for a pessimism utility $U$ which represents $\succcurlyeq$, then there exist canonical parameters $\left(S^{*}, u^{*}\right)$ for $U$ such that $\left|S^{*}\right| \leq|S|$.

Proof. Let $V_{s}(x)=\max _{z \in X} u(z, s)$ for each $x \in X$ and $s \in S$. Then $V_{s}$ is the indirect utility associated with each state $s$. Let $\succcurlyeq_{s}$ stand for the preference represented by $V_{S}$. Let the function $\phi: \mathcal{F}(\succcurlyeq) \rightarrow S$ map each $x \in \mathcal{F}(\succcurlyeq)$ to a state $s \in S$ such that $V_{s}(x)=U(x)$. By the definition of a pessimism utility, such a mapping exists.

We claim that $x \in \mathcal{F}\left(\succcurlyeq_{\phi(x)}\right)$ for every $x \in \mathcal{F}(\succcurlyeq)$. If not, there would exist $x^{\prime} \neq x$ with $x^{\prime} \supset x$ such that $U\left(x^{\prime}\right) \leq V_{s}\left(x^{\prime}\right)=$ $V_{S}(x)=U(x)$ and, by monotonicity, $U\left(x^{\prime}\right)=U(x)$. This in turn would imply $x^{\prime} \neq x, x^{\prime} \supset x$ and $x^{\prime} \sim x$ contradicting $x \in \mathcal{F}(\succcurlyeq)$.

Define the mapping $s^{*}: S \rightarrow 2^{\mathcal{F}(\succcurlyeq)}$ by $s^{*}(s):=\{x \in \mathcal{F}(\succcurlyeq): \phi(x)=s\}$ and let $S^{*}:=\left\{s^{*}(s): s \in S\right\}$. It is easy to see that $\left|S^{*}\right| \leq|S|$.

To see that each $s^{*}(s)$ is a chain in $\mathcal{F}(\succcurlyeq)$, take any pair $x, x^{\prime}$ in $s^{*}(s)$. Then $\phi(x)=s=\phi\left(x^{\prime}\right)$ and $x, x^{\prime} \in \mathcal{F}(\succcurlyeq s)$. Since $V_{s}$ is an indirect utility, Lemma 9 implies $\mathcal{F}\left(\succcurlyeq_{s}\right)$ is a chain. Hence we must have $x \subset x^{\prime}$ or $x \supset x^{\prime}$.

To see that $S^{*}$ covers $\mathcal{F}(\succcurlyeq)$, note that $x \in S^{*}(\phi(x)) \in S^{*}$ for every $x \in \mathcal{F}(\succcurlyeq)$. Hence, $S^{*}$ is a collection of chains in $\mathcal{F}(\succcurlyeq)$ covering $\mathcal{F}(\succcurlyeq)$ and satisfies $\left|S^{*}\right| \leq|S|$. By Lemma 8, there exists $u^{*}: Z \times S^{*} \rightarrow \mathbb{R}$ such that $\left(S^{*}, u^{*}\right)$ are canonical parameters for the pessimism utility $U$.

\section{Proof of Proposition 4}

We proceed with the construction of $S$ and $u: Z \times S \rightarrow \mathbb{R}$ as described in the proof of Theorem 1 in Kreps (1979). Recall that in Kreps' construction $S$ is a subset of $X$ and for each $s \in S$ we have $u(z, s)=a(s)$ if $z \in S$ and $u(z, s)=0$ otherwise, where $a(s)$ are negative numbers. Without loss of generality, we can pick all $a(s)$ to be strictly negative integers. We obtain the representation given in Eq. (2).

The key to the proof is to note that if we replace any state $s_{0} \in S$ with $n$ new states $s_{1}, s_{2}, \ldots, s_{n}$ and for each new state $s_{i}$ and each $z \in Z$ we define $u\left(z, s_{i}\right)=u\left(z, s_{0}\right) / n$, the value of $U(x)$ for any $x \in X$ in Eq. (2) remains unchanged.

Formally, given a state $s_{0} \in S$ and an integer $n \in \mathbb{N}$, for each $i=1, \ldots, n$ define $s_{i}$ to be the pair $\left(s_{0}, i\right)$. Note that $s_{i} \neq s$ for all $s \in S$ and each $i$. Now let $\tilde{S}=\left\{s_{1}, \ldots, s_{n}\right\} \cup S \backslash\left\{s_{0}\right\}$ and define $\tilde{u}: Y \times \tilde{S} \rightarrow \mathbb{R}$ as follows. For each $z \in Y$ and each $s \in S \backslash\left\{s_{0}\right\}$, let $\tilde{u}(z, s)=u(z, s)$ and for each $i=1, \ldots, n$ let $u\left(z, s_{i}\right)=u\left(z, s_{0}\right) / n$.

We thus have for any $x \in X$,

$$
\begin{aligned}
\sum_{s \in \tilde{S}} \max _{z \in X} \tilde{u}(z, s) & =\sum_{s \in S \backslash\left\{s_{0}\right\}} \max _{z \in X} u(z, s)+\sum_{i=1}^{n} \max _{z \in X} \tilde{u}\left(z, s_{i}\right) \\
& =\sum_{s \in S \backslash\left\{s_{0}\right\}} \max _{z \in X} u(z, s)+n \times \max _{z \in X} u\left(z, s_{0}\right) / n \\
& =\sum_{s \in S} \max _{z \in X} u(z, s) \\
& =U(x)
\end{aligned}
$$

so the representation still works with the new set of subjective states $\tilde{S}$ and state dependent utility $\tilde{u}$.

We can interpret this procedure as effectively 'splitting' a subjective state $s_{0}$ into $n$ smaller pieces that, when added together, amount to the original state. In fact, we have

$$
\sum_{i=1}^{n} \max _{z \in X} \tilde{u}\left(z, s_{i}\right)=\max _{z \in X} u\left(z, s_{0}\right)
$$

and all we are doing is substituting the left-hand side for the right-hand side of this equation in the representation (2). Note that this 'splitting' procedure can be applied again to any subjective state in the new representation. The rest of the proof consists in applying this procedure repeatedly.

Since $S \subset X$ is finite, we can partition $S$ into $\sim$-equivalence classes $S_{1}, S_{2}, \ldots, S_{m}$ where for any $z \in S_{j}$ and $z^{\prime} \in S_{k}$ we have $z \succcurlyeq z^{\prime}$ if and only if $j \geq k$. Consider the mapping $s \mapsto j(s)$ where to each $s \in S$ we assign the number $j(s) \in \mathbb{N}$ such that $s \in S_{j(s)}$. We can extend the map $j$ to all of $X$ in the natural way so that $j$ represents $\succcurlyeq$. Note that $j(x) \in \mathbb{N}$ for all $x \in X$ hence the map $x \mapsto-1 / j(x)$ also represents $\succcurlyeq$.

We now proceed to apply the procedure repeatedly: begin with $S_{1}$, the $\sim$-equivalence class of $\succcurlyeq$-least preferred elements of $S$. For each of the original states $s \in S_{1}$ apply the procedure above using $n=|a(s)|$. Note that the value of $n$ will depend on the particular $s \in S_{1}$ and that for all newly created states $s^{\prime}$ and any $z \in Z$ the value of $u\left(z, s^{\prime}\right)$ will be either zero or -1 . 
Now proceed "upwards" through the $\sim$-equivalence classes, applying the splitting procedure to all original states in each equivalence class. Each time the 'splitting' procedure is applied to a state $s \in S_{j}$ choose $n=|a(s)| \times j$ so that the value of $u\left(z, s^{\prime}\right)$ for each newly created state $s^{\prime}$ is either zero or $-1 / j$.

Since applying the 'splitting' procedure does not change the value of $U$, it still represents $\succcurlyeq$. Define $V$ as in Eq. (3) and, with an argument similar to the proof of Proposition 2, it is easy to show that $V(x)=-1 / j(x)$ for all $x \in X$ and therefore $V$ also represents $\succcurlyeq$.

Remark 2. Since in this last proof we effectively split each subjective state $s \in S$ into several new states, the number of subjective states in the representation of Proposition 4 is larger than that of Proposition 2. A modest modification of the proof above maintains the same number of subjective states. Instead of 'splitting' each state into smaller pieces, it consists of 'normalizing' state-dependent utilities so that $u(\cdot, s)$ equals either zero or $-1 / j(s)$ for each $s \in S$ and adding probability weights to the representation in (2) so as to keep the value of $U(x)$ the same for every $x \in X$. We leave the details to the reader.

\section{References}

Ahn, D.S., Sarver, T., 2013. Preference for flexibility and random choice. Econometrica 81 (1), 341-361.

Chatterjee, K., Krishna, R.V., 2011. On preferences with infinitely many subjective states. Econ. Theory 46 (1), 85-98.

Dekel, E., Lipman, B.L., Rustichini, A., 2001. Representing preferences with a unique subjective state space. Econometrica 69 (4), $891-934$.

Dekel, E., Lipman, B.L., Rustichini, A., Sarver, T., 2007. Representing preferences with a unique subjective state space: a corrigendum. Econometrica 75 (2), $591-600$

Dillenberger, D., Postlewaite, A., Rozen, K., 2017. Optimism and pessimism with expected utility. J. Eur. Econ. Assoc. 15 (5), $1158-1175$.

Epstein, L., Marinacci, M., Seo, K., 2007. Coarse contingencies and ambiguity. Theor. Econ. 2 (4), 355-394.

Ergin, H., 2003. Costly Contemplation. Working Paper. MIT.

Ergin, H., Sarver, T., 2010. A unique costly contemplation representation. Econometrica 78 (4), 1285-1339.

Fishburn, P.C., 1970. Utility Theory for Decision-Making. John Wiley and Sons, New York.

Gilboa, I., Schmeidler, D., 1989. Maxmin expected utility with non-unique prior. J. Math. Econ. 18 (2), $141-153$.

Gorno, L., 2016. Additive representation for preferences over menus in finite choice settings. J. Math. Econ. 65, 41-47. doi:10.1016/j.jmateco.2016.05.002.

Harzheim, E., 2005. Ordered Sets. Springer.

Kopylov, I., 2018. Combinatorial Models of Subjective States. Working Paper.

Kreps, D.M., 1979. A representation theorem for "preference for flexibility". Econometrica 47 (3), $565-577$.

Nehring, K., 1999. Preference for flexibility in a savage framework. Econometrica 67 (1), 101-119.

Sadowski, P., 2013. Conditional preference for flexibility: eliciting beliefs from behavior. Theor. Econ. 8 (2), $503-534$

Savage, L.J., 1954. The Foundations of Statistics. John Wiley, New York.

Schenone, P., 2016. Identifying subjective beliefs in subjective state space models. Games Econ. Behav. 95, 59-72. 